\title{
Esophageal Adenosquamous Carcinoma in a Cat
}

\author{
Junko SHINOZUKA ${ }^{1)}$, Hiroyuki NAKAYAMA ${ }^{1)}$, Michio SUZUKI ${ }^{1)}$, Noriko EJIRI ${ }^{1)}$, Koji UETSUKA ${ }^{1)}$, \\ Manabu MOCHIZUKI ${ }^{2)}$, Ryohei NISHIMURA ${ }^{2)}$, Nobuo SASAKI ${ }^{2)}$ and Kunio DOI ${ }^{1) *}$ \\ ${ }^{1)}$ Departments of Veterinary Pathology and ${ }^{2)}$ Veterinary Surgery, Graduate School of Agricultural and Life Sciences, The University of \\ Tokyo, 1-1-1 Yayoi, Bunkyo-ku, Tokyo 113-8657, Japan
}

(Received 5 July 2000/Accepted 6 September 2000)

ABSTRACT. Esophageal carcinoma was observed in an eight-year-old, castrated male, Japanese domestic cat. Histologically, this neoplasm consisted of two different growth patterns, squamous cell carcinoma and adenocarcinoma. The results of immunohistochemical examination supported the fact that the two kinds of neoplastic cells have different characteristics. The tumor was, therefore, diagnosed as adenosquamous carcinoma. Esophageal tumors in the cat are very rare and, if any, neither adenocarcinoma nor adenosquamous carcinoma has been reported up to the present.

KEY WORDS: adenosquamous carcinoma, esophagus, feline.

The esophageal tumors in animals are very rare expect for osteosarcoma and fibrosarcoma in dogs which may be caused by a helminth parasite (Spirocera lupi) [1, 12]. The incidence of the esophageal tumors in cats is less than 0.5 percent of all alimentary tract neoplasms [7]. There are no literatures dealing with primary esophageal carcinoma in cats in Japan. The present report documents a case of esophageal carcinoma in a Japanese domestic cat.

An eight-year-old, castrated male, Japanese domestic cat was presented to the Veterinary Medical Center of the University of Tokyo with complaints of bruxism, dysphasia, occasional vomiting, and regurgitation after eating and drinking. Radiographic examination revealed esophageal constriction at the lower thoracic part and irregular structures at the site. By computed tomography (CT) and esophagoscopic examinations, an intraluminal mass occupying esophageal lumen was found. The patient died due to general weakening.

At necropsy, dilation of the lower portion of the thoracic esophagus just upper the constricted site was found. After opening the esophagus, a white polypoid nodular mass measuring $2 \mathrm{~cm}$ in diameter was noted on the mucosal surface at the site (Fig. 1). The esophageal lumen was occupied by the mass. The cut surface of the mass consisted of grayish white solid tissues and the esophageal wall beneath the mass was thickened (Fig. 2). Neither invasion to the surrounding tissues nor metastasis to other organs was found. No gross lesions were observed in the other organs except for several pulmonary abscesses ( 1 to $3 \mathrm{~cm}$ in diameter) and consolidation in the left anterior lobe.

For light microscopic examination, the esophageal mass was fixed in $10 \%$ neutral buffered formalin and embedded in paraffin by a routine procedure. Four- $\mu \mathrm{m}$-sections were stained with hematoxylin and eosin (HE) or Alcian blue-peri-

\footnotetext{
* CoRrespondence to: DoI, K., Department of Veterinary Pathology, Graduate School of Agricultural and Life Sciences, The University of Tokyo, 1-1-1 Yayoi, Bunkyo-ku, Tokyo 1138657, Japan.
}

odic acid-Schiff (AB-PAS). Immunohistochemical staining was also carried out using formalin-fixed paraffin sections by the avidin-biotin-peroxidase complex method (ABC kit, Vector Laboratories, Burlingame, CA, U.S.A.). The primary antibodies used were rabbit antiserum to human carcinoembryonic antigen (CEA) (DAKO, Carpinteria, CA, U.S.A.) and mouse monoclonal antibody to human keratin NCL-5D3 (BIO-SCIENCE Product, Gerlisstrasse, Switzerland). The latter is known as a glandular epithelial cell marker. For electron microscopic examination, formalin-fixed tissues were refixed in $2.5 \%$ glutaraldehyde and $1 \%$ osmium tetroxide and embedded in Epok 812. Ultrathin sections were double-stained with uranyl acetate and lead citrate and observed using a JEM-1200 EX electron microscope (JEOL, Tokyo, Japan).

Histopathological examination revealed that the neoplastic lesion consisted of two different growth patterns, squamous cell carcinoma and adenocarcinoma (Fig. 3). The former was observed in the luminal polypoid mass, consisting of solid sheets of neoplastic epithelial cells with central

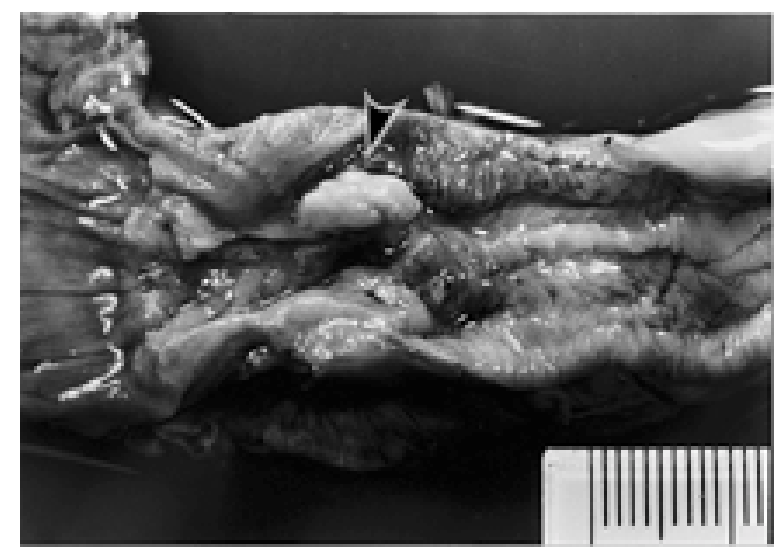

Fig. 1. A neoplastic mass on the esophageal mucosal surface (arrowhead). 


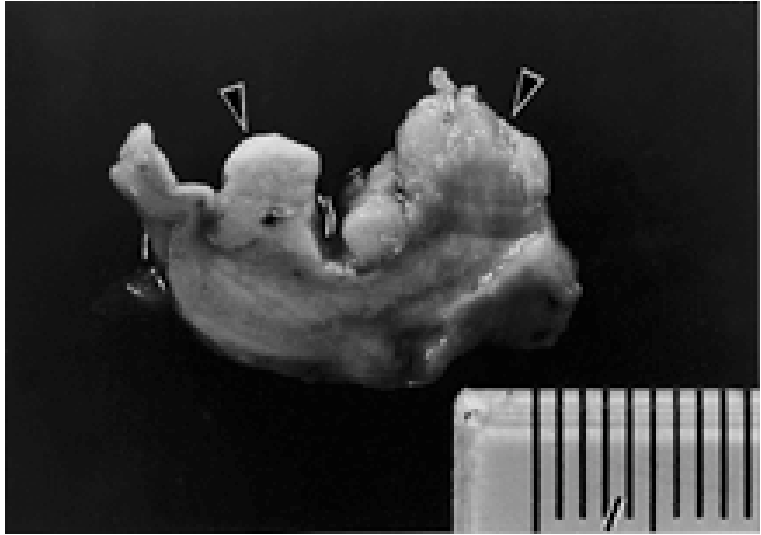

Fig. 2. Cut surface of the esophageal tumor. Polypoid nodules (arrowheads) in the mucosa and thickened esophageal wall.

keratinization or formation of keratin pearls. The cells were polyhedral in shape and uniform in size and have an ovoid vesicular nucleus with one or a few distinct nucleoli. The latter consisting of solid cords or tubules of neoplastic epithelial cells was observed in the submucosal, muscular and serosal layers. The neoplastic cells of adenocarcinoma were cuboidal or polyhedral in shape and irregular in size, possessing an ovoid vesicular nucleus with one or a few distinct nucleoli. In the part of adenocarcinoma, AB-positive secretary materials were shown in the tubular lumen or within the cytoplasm of the tumor cells. Numerous mitotic figures were observed in both tumor lesions. Neither invasion nor metastasis of the tumor to other tissues was detected even through histopathological observation.

Tubular and glandular neoplastic cells were positively stained with anti-keratin NCL-5D3 antibody while neoplastic squamous cells and normal esophageal epithelial cells were negative. Only the luminal surface of tubular and glandular neoplastic cells was positive for CEA.

Ultrastructural observation revealed cytoplasmic tonofilament-like fibrils and intercellular desmosome-like structures in the squamous cell carcinoma lesion and microvilli-like structures on the luminal surface of the glandular neoplastic cells. In cells constructing the glandular structure, cytoplasm was rich in rough endoplasmic reticulum and ribosome, and secretary granules surrounded by a single membrane were
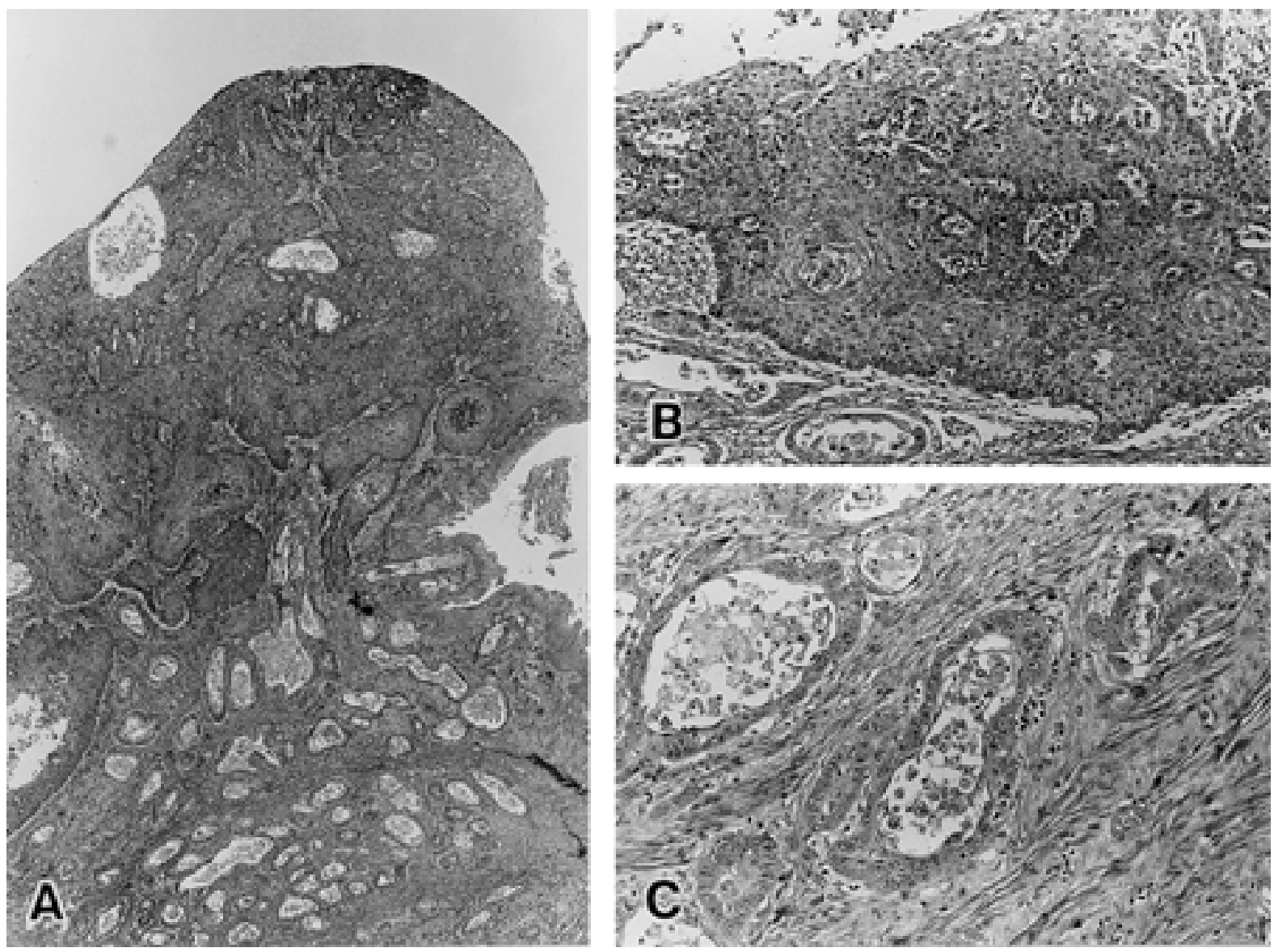

Fig. 3. Histology of the esophageal tumor. A: The tumor consists of squamous (upper) and tubular/glandular (lower) parts. HE stain, $\times$ 25. B: Part of squamous cell carcinoma. HE stain, $\times 40$. C: Part of adenocarcinoma. HE stain, $\times 120$. 
also seen.

The esophageal neoplasm of the present case consisted of two different growth patterns, squamous cell carcinoma and adenocarcinoma, and therefore was diagnosed as adenosquamous carcinoma. The immunohistochemical examination for the present case supported the fact that the two kinds of neoplastic epithelial cells have different characteristics. Neither adenocarcinoma nor adenosquamous carcinoma in cats has been reported up to the present. Adenosquamous carcinoma of the esophagus in man is also very rare [2].

According to the previous reports, esophageal tumors in cats, such as squamous cell carcinoma [3, 9], lymphosarcoma [3] and neuroendocrine carcinoma [10], were observed particularly in old castrated males. Clinical signs of esophageal neoplasms are chronic obstructive esophageal symptoms, including anorexia, depression, vomiting, weight loss, pain after food intake, systemic emaciation, dysphasia, regurgitation and respiratory disturbances [13]. Aspiration pneumonia due to regurgitation was the most crucial respiratory lesion. The above-mentioned symptoms were similar in the present case. Pulmonary abscesses and pneumonia observed at necropsy may be the direct cause of death. Metastasis to the caudal cervical, mediastinal and bronchial lymph nodes, lungs, kidneys, thyroid glands, and spleen has been described in the previous feline case of esophageal squamous cell carcinoma [7], but no metastasis was observed in the present case.

The cause of esophageal neoplasms in cats is still unknown, but certain environmental carcinogens have been incriminated. Cotchin [4] reported a frequent occurrence of feline esophageal carcinoma in London and suggested that the very high frequency was due to ingestion of an unknown carcinogen by self-grooming. There are several opinions on the histogenesis of esophageal adenocarcinoma in man. One is ascribed to submucosal glands and ducts, and others to glandular metaplasia of squamous cell carcinoma, or to Barrett's esophagus $[6,8,11]$. However, the present feline tumor arose in the lowest third of the esophagus where esophageal glands are not observed in cats. The esophageal glands are present only at the pharyngoesophageal junction in cats [5], which was confirmed by our examinations with normal cats (data not shown). Therefore, the second hypothesis can be excluded. Barrett's esophagus is associated with an increased risk of human esophageal adenocarcinoma [6], but that in cats is still obscure.

Accordingly, it is most probable that the present esophageal adenosquamous carcinoma may be developed through the process of glandular metaplasia of squamous cell carcinoma that had initially resided.

\section{REFERENCES}

1. Bailey, W. S. 1979. J. Am. Vet. Med. Assoc. 175: 148-50.

2. Bomb, J. A., Riverola, A., Bordas, J. M. and Cardesa, A. 1991. Pathol. Res. Prac. 187: 514-519.

3. Cotchin, E. 1959. Vet. Rec. 71: 1040-1054.

4. Cotchin, E. 1966. Ann. R.Coll. Surg. Engl. 38: 92-116.

5. Dellmann, H. D. and Brown, E. M. 1981. pp. 220-223 In: Textbook of Veterinary Histology, 2nd ed., Lea \& Febriger, Philadelphia, U.S.A.

6. Haggitt, R. C. 1994. Human Pathol. 25: 982-993.

7. Happe, R. P., Wolvekamp, W. T. and Toorenburg, J. 1978. Tijdschr. Diergeneeskd. 103: 1080-1086.

8. Kuwano, H., Nagamatsu, M., Ohno, S., Matsuda, H. and Mori, M. 1988. Cancer 62: 1568-1572.

9. Morton, J. E. 1980. pp. 381-382. In: Tumors in Domestic Animals, 3rd ed., University of California Press, Berkeley and Los Angeles, California, U.S.A.

10. Patnaik, A. K., Erlandson, R. A. and Lieberman, P. H. 1990. Vet. Pathol. 27: 128-130.

11. Pascal, R. R. and Clearfield, H. R. 1987. Dig. Dis. Sci. 32: 428432.

12. Ridway, R. L. and Suter, P. F. 1979. J. Am. Vet. Med. Assoc. 174: 700-704.

13. Vernon, F. F. and Roudebush, P. 1980. J. Am. Anim. Hosp. Assoc. 16: 547-550. 